lustitia Socialis. Revista Arbitrada de Ciencias Jurídicas.

Año V. Vol. V. №3. Edición Especial. 2020-III:

Universidad Regional Autónoma de los Andes

Hecho el depósito de Ley: FA2016000064

ISSN: 2542-3371

FUNDACIÓN KOINONIA (F.K). Santa Ana de Coro, Venezuela

Laura Alicia Colcha-Ramos; Luis Renato Daqui-Orozco; Gisella Lizbeth Urquizo-Padilla

http://dx.doi.org/10.35381/racii.v5i3.1085

\title{
Principio de Celeridad en los Procesos Judiciales durante pandemia covid-19 en Riobamba
}

\section{Principle of speed in judicial proceedings during the covid-19 pandemic in Riobamba}

\author{
Laura Alicia Colcha-Ramos \\ aliciacolchar@hotmail.com \\ Universidad Regional Autónoma de los Andes, Riobamba \\ Ecuador \\ https://orcid.org/0000-0002-4805-7271 \\ Luis Renato Daqui-Orozco \\ dr.luisrdo22@uniandes.edu.ec \\ Universidad Regional Autónoma de los Andes, Riobamba \\ Ecuador \\ Gisella Lizbeth Urquizo-Padilla \\ dr.gisellalup18@uniandes.edu.ec \\ Universidad Regional Autónoma de los Andes, Riobamba \\ Ecuador
}

Recibido: 31 de octubre de 2020

Revisado: 10 de octubre de 2020

Aprobado: 05 de diciembre de 2020

Publicado: 10 de diciembre de 2020 
lustitia Socialis. Revista Arbitrada de Ciencias Jurídicas.

Año V. Vol. V. N³. Edición Especial. 2020-III:

Universidad Regional Autónoma de los Andes

Hecho el depósito de Ley: FA2016000064

ISSN: 2542-3371

FUNDACIÓN KOINONIA (F.K). Santa Ana de Coro, Venezuela

Laura Alicia Colcha-Ramos; Luis Renato Daqui-Orozco; Gisella Lizbeth Urquizo-Padilla

\title{
RESUMEN
}

La investigación tiene por objetivo principal analizar el principio de celeridad en los procesos judiciales durante pandemia por covid-19 en la ciudad de Riobamba, ubicada en el Cantón Riobamba de la Provincia de Chimborazo - Ecuador. Se generó primordialmente desde el método analítico - sintético, trabajándose en evidencias y testimonios documentados sobre el principio de celeridad en los procesos judiciales durante pandemia por covid-19 en la ciudad de Riobamba. Se ha determinado que durante los meses de confinamiento ha existido un incremento en los índices de denuncias y delitos, entre los más comunes tenemos la violencia en contra de la mujer. El principio de celeridad ha jugado un papel importante y se ha aplicado eficazmente dentro de los procesos de carácter judicial en la ciudad de Riobamba, debido a que se han logrado resultados positivos en favor de la justicia.

Descriptores: Derecho a la justicia; administración de justicia; aplicación de la ley. (Palabras tomadas del Tesauro UNESCO).

\begin{abstract}
The main objective of the research is to analyze the principle of speed in judicial processes during a COVID-19 pandemic in the city of Riobamba, located in the Riobamba Canton of the Chimborazo Province - Ecuador. It was generated primarily from the analytical synthetic method, working on evidence and documented testimonies on the principle of speed in judicial processes during the COVID-19 pandemic in the city of Riobamba. It has been determined that during the months of confinement there has been an increase in the rates of complaints and crimes, among the most common we have violence against women. The principle of speed has played an important role and has been effectively applied within judicial processes in the city of Riobamba, due to the fact that positive results have been achieved in favor of justice.
\end{abstract}

Descriptors: Right to justice; administration of justice; law enforcement. (Words taken from the UNESCO Thesaurus). 
lustitia Socialis. Revista Arbitrada de Ciencias Jurídicas.

Año V. Vol. V. N³. Edición Especial. 2020-III:

Universidad Regional Autónoma de los Andes

Hecho el depósito de Ley: FA2016000064

ISSN: 2542-3371

FUNDACIÓN KOINONIA (F.K). Santa Ana de Coro, Venezuela

Laura Alicia Colcha-Ramos; Luis Renato Daqui-Orozco; Gisella Lizbeth Urquizo-Padilla

\section{INTRODUCCIÓN}

La administración de justicia en el Ecuador, durante la pandemia por COVID-19 ha sufrido cambios drásticos, alterándose su normal funcionamiento, decidiéndose cerrar los juzgados, facilitar plazos procesales y dar otra fecha para la realización de juicios todo esto con el fin de evitar que se propague el COVID 19 y poner en riesgo a los servidores judiciales (García-Mejía, 2020). Esto conllevó a la adopción de otras formas de llevar a cabo la justicia, es así que se implementan las teleaudiencias por medio de herramientas tecnológicas y del internet. En este sentido, la tecnología ha intervenido de una manera significante en estos momentos difíciles, recurriéndose a la misma para tratar de solucionar los problemas o inconvenientes causados por el confinamiento.

De esta manera como manifiesta (García-Mejía, 2020), las audiencias que se llevan a cabo vía Internet poseen un reglamento, así como las audiencias comunes, por lo que se debe respetar las mismas para que el proceso sea eficaz; el Estado Ecuatoriano ha adoptado estos sistemas para no parar el funcionamiento de juzgados, fiscalía, entre otras entidades judiciales.

En este se sentido, existen protocolos y reglas que se deben tomar para intervenir en los procesos, audiencias se debe tener una conectividad a Internet estable, se realizarán dichos procesos como tradicionalmente se han desarrollado presencialmente, los sujetos procesales se deberán presentar, los defensores técnicos de cada parte deben portar la documentación y la vestimenta correcta para intervenir en dichos procesos, se debe regular cámaras, micrófonos para su intervención y no haya ningún tipo de dificultad en su desenvolvimiento (García-Mejía, 2020).

Desde el punto de vista de (García-Mejía, 2020), la emergencia sanitaria parece haber tenido resultados positivos en lo que tiene que ver con la aplicación de la Justicia, la nueva normalidad judicial, términos utilizados en los últimos meses, demuestra que los nuevos sistemas empleados mediante vías tecnológicas aportan celeridad a los juicios y la digitalización permite la continuidad del servicio. En este caso, "la pandemia ha 
lustitia Socialis. Revista Arbitrada de Ciencias Jurídicas.

Año V. Vol. V. N³. Edición Especial. 2020-III:

Universidad Regional Autónoma de los Andes

Hecho el depósito de Ley: FA2016000064

ISSN: 2542-3371

FUNDACIÓN KOINONIA (F.K). Santa Ana de Coro, Venezuela

Laura Alicia Colcha-Ramos; Luis Renato Daqui-Orozco; Gisella Lizbeth Urquizo-Padilla

resultado un catalizador positivo", por cuanto se han agilizado todo lo que tiene que ver con los procesos judiciales.

El 9 de abril de 2020 la Corte Interamericana de Derechos Humanos, declara sobre el COVID-19 y derechos humanos, alude problemas y desafíos a atender con perspectiva de derechos humanos, adoptando las obligaciones internacionales manifiesta que la aplicación de justicia es indispensable, por cuanto se debe proteger los derechos que manifiestan en la Constitución, si no se cumplen dichos mandatos las defensoras y defensores de derechos humanos, estarán evaluando las funciones del Sistema Judicial y su celeridad (Corte Interamericana de Derechos Humanos, 2020).

Se reitera que cuando llegó la pandemia al Ecuador, durante un tiempo se mantuvieron cerrados los tribunales, de esta manera se llegó a observar que estos son de gran importancia para millones de personas, debido que la mayoría de los ciudadanos acuden a estos lugares para poder llegar a resolver sus problemas, de esta forma se podría llegar a la conclusión de como impartir justicia, por cuanto es un tema esencial para tratar (Carbonell, 2020).

La pandemia del COVID-19 llegó a América Latina en circunstancias de modernización en los poderes judiciales, cabe recalcar que los juicios orales que se han dado durante mucho tiempo se han llegado a adquirir muchos beneficios tanto en ganancias de eficacia como celeridad, un gran ejemplo es Ecuador ya que pasar de procesos escritos a orales llegó a reducir un 25 \% en tiempo de duración de los juicios (García-Mejía, 2020).

También el avance y el uso de la tecnología ha llegado a ser de gran importancia, ya que de esta manera los expedientes pueden ser digitales para los juicios y dentro de la gestión administrativa, ha sido de gran beneficio, de esta manera existe mayor acceso remoto dentro de la justicia, aunque esta nueva forma de modernización es de gran beneficio aun algunas regiones o países no se acogen y lo hacen de manera escrita los juicios (García-Mejía, 2020). 
lustitia Socialis. Revista Arbitrada de Ciencias Jurídicas.

Año V. Vol. V. N³. Edición Especial. 2020-III:

Universidad Regional Autónoma de los Andes

Hecho el depósito de Ley: FA2016000064

ISSN: 2542-3371

FUNDACIÓN KOINONIA (F.K). Santa Ana de Coro, Venezuela

Laura Alicia Colcha-Ramos; Luis Renato Daqui-Orozco; Gisella Lizbeth Urquizo-Padilla

Anteriormente se observaba que en los despachos las carpetas se encontraban amontonadas en los escritorios, actualmente ya no se da ese tipo de cosas, por cuanto la totalidad de los archivos se encuentran disponibles en el bando de datos durante las 24 horas al día, siendo de gran ayuda y más efectiva de adquirir a aquellos documentos (Muniz, 2020).

Durante esta pandemia se ha llegado observar también que la justicia dentro de los juicios telemáticos no está preparada para adoptarse a esta nueva forma de realizar un proceso judicial sin poner en riesgo los derechos de los ciudadanos, aunque de cierta manera los abogados lograron intervenir en su ámbito de trabajo haciéndolo con responsabilidad y con la ayuda del Estado (Robledo, 2020).

Utilizar la tecnología para los procesos judiciales han sido de cierta de manera de gran ayuda ya que el contagio del COVID-19 se llega prologar de manera rápida y hasta el momento transcurrido no han llegado a obtener una cura a nivel mundial siendo así de gran importancia adquirir estas medidas de aislamiento para evitar el contagio de tal virus que es invisible (Espeche, 2020).

EI Consejo de la Judicatura llego aprobar un procedimiento para llegar a emitir con mayor celeridad las boletas de libertad y otros beneficios penitenciarios para las personas que se encuentran con privación de libertad por la emergencia sanitaria del COVID.19 dentro del Estado Ecuatoriano, un ejemplo es que los jueces de garantías penitenciarias puedan emitir boletas si la necesidad de convocar a audiencia (Puente, 2020).

Dentro de los juzgados la gente se entiende hablando, la oralidad en los juicios busca que los afectados se encuentre en una sala, el juez revisara todas las pruebas y escuchara los diferentes argumentos que tiene cada parte para así poder llegar a una conclusión y dictar la sentencia, de esta manera el proceso se da de manera rápida con máxima transparencia (García-Mejía, 2020).

El medio para la impartición de poder es el sistema procesal, las normas se sustentan en aquellos principios de simplificación, eficacia, celeridad, mediación, uniformidad y economía procesal para que de esta manera se llegue hacer efectiva las garantías del 
lustitia Socialis. Revista Arbitrada de Ciencias Jurídicas.

Año V. Vol. V. N³. Edición Especial. 2020-III:

Universidad Regional Autónoma de los Andes

Hecho el depósito de Ley: FA2016000064

ISSN: 2542-3371

FUNDACIÓN KOINONIA (F.K). Santa Ana de Coro, Venezuela

Laura Alicia Colcha-Ramos; Luis Renato Daqui-Orozco; Gisella Lizbeth Urquizo-Padilla

debido proceso, el cuerpo de principios constituye un sistema en sí y todos aquellos principios tienen una importancia fundamental en su contenido para el ordenamiento jurídico (Jarama-Castillo, 2019, p. 315).

En razón de lo planteado, la investigación se justifica por cuanto se aborda una temática que en el pasado reciente ha concebido problemas de celeridad, produciéndose dilaciones en el proceso judicial, siendo que ahora se maneja en modalidad virtual los juicios, se corre el riesgo de que sean trasladados los vicios y problemas de retardo procesal, siendo esto contrario al principio universal de celeridad de la justicia (JaramaCastillo, 2019), lo cual concuerda con la posición de (Canelo-Rabanal, 2006), quien indica que el proceso de celeridad no es concepto ambiguo o abstracto, se genera en la realidad en concordancia con una praxis judicial en respuesta a las necesidades de los afectados, ante lo cual, se encuentra constantemente ante desafíos, siendo uno de ellos, el ser efectiva en la atención judicial durante el confinamiento por COVID - 19 en el Ecuador. En síntesis, la investigación tiene por objetivo principal analizar el principio de celeridad en los procesos judiciales durante pandemia por covid-19 en la ciudad de Riobamba, ubicada en el Cantón Riobamba de la Provincia de Chimborazo - Ecuador.

\section{MÉTODOS}

El trabajo investigativo se generó primordialmente desde el método analítico - sintético, trabajándose en evidencias y testimonios documentados sobre el principio de celeridad en los procesos judiciales durante pandemia por covid-19 en la ciudad de Riobamba, ubicada en el Cantón Riobamba de la Provincia de Chimborazo - Ecuador, ubicándose información que configuró un análisis de las partes heurísticas referidas en la documentación intervenida, estructurándose un aporte teórico en razón de la información reconstruida (Lopera-Echavarría, et al., 2010).

Apoyándose metodológicamente en un tipo de investigación descriptiva documental con diseño bibliográfico, aplicándose la técnica de análisis de contenido para extraer las ideas 
lustitia Socialis. Revista Arbitrada de Ciencias Jurídicas.

Año V. Vol. V. N³. Edición Especial. 2020-III:

Universidad Regional Autónoma de los Andes

Hecho el depósito de Ley: FA2016000064

ISSN: 2542-3371

FUNDACIÓN KOINONIA (F.K). Santa Ana de Coro, Venezuela

Laura Alicia Colcha-Ramos; Luis Renato Daqui-Orozco; Gisella Lizbeth Urquizo-Padilla

centrales con la finalidad de ser compuestas sintéticas en un resultados y conclusiones de estudio.

\section{RESULTADOS}

La aplicación de la justica a través de mecanismos basados en la internet en sustitución de los juicios presenciales a causa de la pandemia por COVID-19, es una innovación en el sistema judicial ecuatoriano, por lo tanto, es necesario medir su aplicación con la finalidad de adoptar la medidas para fortalecer tal perspectiva, en este sentido, el Consejo de la Judicatura (2020), expresa que "la implementación de herramientas telemáticas como la Oficina de Gestión Judicial Electrónica, componente del e-SATJE 2020, que ha permitido a los abogados, fiscales, defensores públicos y servidores judiciales gestionar procesos judiciales digitalmente, de manera rápida, segura y transparente" (p. 1).

Puede resumirse que el tiempo de los procesos judiciales se ha mejorado sustancialmente con la implementación de las tecnologías ya que se puede evitar largas filas. La Justicia se vuelve más transparente ya que al digitalizarla la justicia mejora y hay menos posibilidades de casos de corrupción en el Sistema judicial. La Justicia se vuelve más transparente ya que al digitalizarla la justicia mejora y hay menos posibilidades de casos de corrupción en el Sistema judicial. Las etapas de los procesos que se realizan frente al juez y debe cumplir una serie parámetros y debe contar con las formalidades del caso y este tiempo de espera es el que retarda la resolución definitiva del caso.

Antes de la Pandemia la escasez de recursos, la ausencia de proyectos, el elevado número de audiencias fallidas y una elevada carga procesal, como la razón inicial de la problemática en el sistema de justicia y su celeridad, ya que debido a estos problemas no se avanzaban los procesos. Tiempo antes de ser declarado el estado de emergencia decretado el sistema de justicia y sus procesos eran lentos. Anterior a la pandemia se han presentado casos de corrupción en el sistema judicial. Muchas veces se han retardado los procesos por malos administradores de justicia. De la forma que la pandemia llegó a Ecuador su sistema judicial y procesos se realizaban juicios orales y 
lustitia Socialis. Revista Arbitrada de Ciencias Jurídicas.

Año V. Vol. V. N³. Edición Especial. 2020-III:

Universidad Regional Autónoma de los Andes

Hecho el depósito de Ley: FA2016000064

ISSN: 2542-3371

FUNDACIÓN KOINONIA (F.K). Santa Ana de Coro, Venezuela

Laura Alicia Colcha-Ramos; Luis Renato Daqui-Orozco; Gisella Lizbeth Urquizo-Padilla

esto traía como consecuencia positiva la celeridad de los procesos y a la vez las audiencias o dichos procesos se revestían de eficiencia en su realización, de esta manera se evidenció un cambio en comparación cuando en el país se empleaban los procesos escritos, los cuales eran demorados y al pasar a la forma oral (virtual) se redujo en un $25 \%$ el tiempo de duración de los juicios.

\section{DISCUSIÓN}

Como punto primordial se destaca el total desacuerdo con los casos en los cuales se ha mostrado un índice de corrupción dentro de la justicia, ya que como ciudadanos se procura tener la plena confianza en el sistema de justicia, sin embargo, al existir corrupción o retardo procesal, se viola el principio de celeridad, generándose una opinión de desconfianza sobre quienes aplican la justicia, desprestigiándose, dando paso así, a prácticas contrarias a la ética en el manejo de dictaminar sentencia (Ramos, 2017).

Por lo tanto, se apoya que el principio de la celeridad sea aplicado con total plenitud dentro de los procesos y con mucha más razón en estos tiempos de confinamiento que estamos atravesando a causa de la pandemia del COVID-19, ya que todo ha llegado a entrar en un plan tecnológico, lo cual concuerda con la postura del futuro de la justicia, propuesto por (García-Barrera, 2018), de contar con documentos electrónicos y juicios meramente electrónico a través de la tecnología de la información y comunicación TIC. El uso de las TIC en el sistema de justicia en procura de poner en práctica el principio de celeridad evita que se queden estancados los juicios procesales, por cuanto estos al pasar de los días pueden llegar a ocasionar varios conflictos, ya que de cierta manera es donde se llegan a resolver los casos y el juez brinde solución contribuyendo en mantener la sociedad en armonía, generándose así una renovada gestión pública (Ávila-Barrios, 2014).

Se ha determinado que durante los meses de confinamiento ha existido un incremento en los índices de denuncias y delitos, entre los más comunes tenemos la violencia en contra de la mujer, es en este tipo de casos en los que se ha visto una gran necesidad 
lustitia Socialis. Revista Arbitrada de Ciencias Jurídicas.

Año V. Vol. V. N³. Edición Especial. 2020-III:

Universidad Regional Autónoma de los Andes

Hecho el depósito de Ley: FA2016000064

ISSN: 2542-3371

FUNDACIÓN KOINONIA (F.K). Santa Ana de Coro, Venezuela

Laura Alicia Colcha-Ramos; Luis Renato Daqui-Orozco; Gisella Lizbeth Urquizo-Padilla

del actuar de la justicia con celeridad, para buscar que estos casos sean atendidos a la brevedad posible y que los mismos no queden en la impunidad, ya que esas es la finalidad del principio de celeridad dentro de la justicia (Montero-Medina, et al., 2020).

Por lo tanto, se comprende que el uso de la tecnología ya no es considerado como una opción, sino, como una obligación para prevenir la propagación del COVID-19, es por ello que el uso tecnológico ha ido abarcando a toda la sociedad, desde estudiantes, pequeños emprendedores, hasta profesionales y grandes empresarios. Esto ha traído una división de posturas, hay quienes están a favor y quienes demuestran inconformidad con estas medidas adoptadas, los que están a favor tienen como respaldo que es la única manera de continuar con sus planes de surgimiento y los que no la apoyan timen como argumento que esta modalidad no presta garantías de efectividad en las labores (Garat, et al., 2020).

\section{CONCLUSIONES}

Se han presentado cambios totalmente radicales en el cotidiano vivir no solo en nuestra localidad sino también a nivel provincial, nacional e internacional. Estos cambios han abarcado a todas las esferas de la sociedad, y dentro de ellas está la justicia, haciendo que se busque una manera suplementaria de atención y aplicación de la misma, viendo obligados a los profesionales del derecho a implementar nuevos planes de trabajo para la obtención de resultados positivos dentro de sus labores. Es así como se ha dado un realce al principio de celeridad, de esta forma se busca evitar que ocurran casos de corrupción dentro de los procesos y que los procedimientos de los mismos sean llevados de manera correcta por medio de la Jueza o Juez competente.

La importancia que tiene la Justicia en esta época de pandemia es imprescindible ya que no por estar en emergencia sanitaria no se puede detener dicho sistema, esta tarea de relevancia que el estado democrático le atribuye al poder judicial implica el cumplimiento de una serie de obligaciones inexcusables por parte de los hombres que desempeñan funciones en tribunales, juzgados, y en la administración de justicia en general, tales como: conocer en detalle los casos que llegan a su competencia y decidir sobre los 
lustitia Socialis. Revista Arbitrada de Ciencias Jurídicas.

Año V. Vol. V. N³. Edición Especial. 2020-III:

Universidad Regional Autónoma de los Andes

Hecho el depósito de Ley: FA2016000064

ISSN: 2542-3371

FUNDACIÓN KOINONIA (F.K). Santa Ana de Coro, Venezuela

Laura Alicia Colcha-Ramos; Luis Renato Daqui-Orozco; Gisella Lizbeth Urquizo-Padilla

mismos de manera fundada y siempre en concordancia con el derecho, en el momento que corresponde, porque la justicia tardía no será justicia y de cierta forma si la justicia se detiene por la pandemia todo el Estado lo hace ya que no se podría ni juzgar ni sancionar.

No se debe permitir que los abogados sí están llevando a cabo a marchas forzosas mediante una transformación digital deje fuera a las instituciones. La pandemia no puede ser motivo para dejar aún más desprotegidos a los ecuatorianos, nuestro Estado al ser garantista en derechos, nos señala en nuestra Constitución la protección para todas y todos los ciudadanos y con mucha más razón a quienes conforman y son parte de la población vulnerable en nuestro país.

Se puede evidenciar en la tutela efectiva de las juezas, jueces y en los tribunales el principio de celeridad al momento en que se garantiza a los ciudadanos en el ejercicio pleno de sus derechos y de sus propios intereses de legitimidad y que en ninguno de los mencionados casos se vea afectada la defensa, protección o ayuda que la misma celeridad garantiza para todas las personas.

Durante la pandemia del COVID-19 varios de los profesionales del derecho han elegido apegarse a la tecnología, siendo este un instrumento de gran ayuda para contrarrestar lo que estamos viviendo, llevándose a cabo de esta manera, consultas, audiencias, asesoramientos, etc. lo mismo que ha servido para que se pueda cumplir con el principio de celeridad al momento de aplicar la justicia, pero lamentablemente algunos de ellos no han 'presentado estar totalmente capacitados y ello denota que no hay una adaptación al momento de ejercer sus conocimientos sin poner en riesgo los derechos de los ciudadanos.

La celeridad es de suma importancia por cuanto este es un principio en el cual se garantiza que el proceso judicial se dará de manera rápida y eficaz, es necesario enfocarnos sobre este tema, ya que durante esta pandemia del COVID-19, la celeridad de los procesos judiciales que se encontraban estancados o paralizados durante mucho tiempo, gracias a la tecnología lograron tomar medidas necesarias para llegar a 
lustitia Socialis. Revista Arbitrada de Ciencias Jurídicas.

Año V. Vol. V. N³. Edición Especial. 2020-III:

Universidad Regional Autónoma de los Andes

Hecho el depósito de Ley: FA2016000064

ISSN: 2542-3371

FUNDACIÓN KOINONIA (F.K). Santa Ana de Coro, Venezuela

Laura Alicia Colcha-Ramos; Luis Renato Daqui-Orozco; Gisella Lizbeth Urquizo-Padilla

solucionar esos problemas, el debido proceso es necesario para la existencia de la justicia, de esta manera se obtendrá armonía dentro de la sociedad, llegar a resolver los procesos judiciales virtualmente para evitar contagios ya que este COVID-19 se prolonga de forma rápido pero también puede llegar a ocasionar algunas dificultades ya que no es lo mismo defender un caso presencial que virtual

En Riobamba en los últimos meses del año 2020 se ha evidenciado una clara celeridad en la justicia, tanto Fiscalía y los Juzgados respectivamente han empleado los métodos tecnológicos para evitar contagios de COVID 19 en funcionarios judiciales se han resuelto casos que se han estado investigando hasta la fecha, se han concluido varios juicios y se ha dictado sentencia a los implicados.

El principio de celeridad ha jugado un papel importante y se ha aplicado eficazmente dentro de los procesos de carácter judicial en la ciudad de Riobamba, debido a que se han logrado resultados positivos en favor de la justicia, por cuanto el mismo ha evitado que los procesos se acumulen y pasen sin ser atendidos de la manera correcta como verdaderamente deberían ser llevados, respetando el debido proceso y sin postergación, así también se evita que varios de los procesos queden en la impunidad por no haber sido atendidos en el tiempo correcto como ya ha ocurrido anteriormente.

\section{FINANCIAMIENTO}

No monetario.

\section{AGRADECIMIENTO}

A la Universidad Regional Autónoma de Los Andes; por motivar el desarrollo de la Investigación. 
lustitia Socialis. Revista Arbitrada de Ciencias Jurídicas.

Año V. Vol. V. N³. Edición Especial. 2020-III:

Universidad Regional Autónoma de los Andes

Hecho el depósito de Ley: FA2016000064 ISSN: 2542-3371

FUNDACIÓN KOINONIA (F.K). Santa Ana de Coro, Venezuela

Laura Alicia Colcha-Ramos; Luis Renato Daqui-Orozco; Gisella Lizbeth Urquizo-Padilla

\section{REFERENCIAS CONSULTADAS}

Ávila-Barrios, D. (2014). El uso de las TICs en el entorno de la nueva gestión pública mexicana. [The use of ITC's in the Vicinity of New Public Management Mexican]. Andamios, 11(24), 263-288.

Canelo-Rabanal, R. V. (2006). La celeridad procesal, nuevos desafíos. Hacia una reforma integral del proceso civil en busca de la justicia pronta. [The procedural speed, new challenges. Towards a comprehensive reform of the civil process in search of prompt justice]. Revista Iberoamericana de Derecho Procesal Garantista. Recuperado de https://n9.cl/5ew3a

Carbonell, M. (2020). Justicia y pandemia. [Justice and pandemic]. [Internet]. Recuperado de https://n9.cl/jxh7c

Consejo de la Judicatura (2020). Prestación del servicio de justicia en Ibarra se cumple bajo estrictas medidas de bioseguridad. [Provision of the justice service in lbarra is carried out under strict biosecurity measures]. Recuperado de https://n9.cl/a13os

Corte Interamericana de Derechos Humanos (2020). COVID-19 y derechos humanos: los problemas y desafíos deben ser abordados con perspectiva de derechos humanos y respetando las obligaciones internacionales. [COVID-19 and human rights: problems and challenges must be approached with a human rights perspective and respecting international obligations]. Recuperado de https://n9.cl/2x2h

Espeche. S. (2020). Justicia y tecnología en el contexto de la pandemia del COVID-19. [Justice and technology in the context of the COVID-19 pandemic]. [Internet]. Recuperado de https://n9.cl/4ris

Garat, M. P., Risso Ferrand, M., Ruocco, G., Ramos Cabanellas, B., Valentín, G., Slinger, L., Costa, L., Herdt, C., Gamarra, D., \& Guerra, W. (2020). El Derecho frente a la pandemia por COVID-19. [The Law in the face of the COVID-19 pandemic]. Revista De Derecho, (21), 01-24. https://doi.org/10.22235/rd.vi21.2193

García-Barrera, M. (2018). Juzgado sin papel, un paso más de la justicia electrónica [Paperless Courts, One More Step of Electronic Justice]. Revista IUS, 12(41), 133154.

García-Mejía, M. (2020). Justicia y COVID-19: 3 formas de impartir justicia durante una pandemia. [Justice and COVID-19: 3 Ways to Deliver Justice During a Pandemic]. [[Internet]. Recuperado de https://n9.cl/f06z 
lustitia Socialis. Revista Arbitrada de Ciencias Jurídicas.

Año V. Vol. V. N³. Edición Especial. 2020-III:

Universidad Regional Autónoma de los Andes

Hecho el depósito de Ley: FA2016000064 ISSN: 2542-3371

FUNDACIÓN KOINONIA (F.K). Santa Ana de Coro, Venezuela

Laura Alicia Colcha-Ramos; Luis Renato Daqui-Orozco; Gisella Lizbeth Urquizo-Padilla

Jarama-Castillo, Z, Vásquez-Chávez, J, \& Durán-Ocampo, A. (2019). El principio de celeridad en el código orgánico general de procesos, consecuencias en la audiencia. [The principle of speed in the general organic code of processes, consequences for the audience]. Revista Universidad y Sociedad, 11(1), 314-323.

Lopera-Echavarría, J, \& Ramírez-Gómez, C, \& Zuluaga-Aristazábal, M, \& Ortiz-Vanegas, J. (2010). El método analítico como método natural. Nómadas. Critical Journal of Social and Juridical Sciences, 25(1).

Montero-Medina, D., Bolívar-Guayacundo, M., Aguirre-Encalada, L., \& MorenoEstupiñán, A. (2020). Violencia intrafamiliar en el marco de la emergencia sanitaria por el COVID-19. [Domestic violence in the framework of the health emergency caused by COVID-19]. CienciAméRica, 9(2), 261-267.

Muniz, M. (2020). Justicia durante la cuarentena: tecnología es aliada del trámite judicial. [Justice during quarantine: technology is an ally of the judicial process]. [Internet]. Recuperado de https://n9.cl/jxh7c

Puente. D. (2020). Judicatura busca acelerar trámites de libertad para presos durante la emergencia sanitaria. [Judiciary seeks to speed up procedures for the release of prisoners during the health emergency]. Recuperado de https://n9.cl/n1w0

Ramos, R. (2017). Derecho constitucional en ecuador y aspectos sobre la corrupción desde lo penal. [Constitutional law in ecuador and semblances on the corruption from penal's view]. Revista Chakiñan de Ciencias Sociales y Humanidades, (2), 35-46.

Robledo. J. (2020). En estos momentos, la Justicia no está preparada para adaptarse a las nuevas tecnologías. [At this time, Justice is not prepared to adapt to new technologies]. [Internet]. Recuperado de https://n9.cl/f1zd8

(C2020 por los autores. Este artículo es de acceso abierto y distribuido según los términos y condiciones de la licencia Creative Commons Atribución-NoComercial-Compartirlgual 4.0 Internacional (CC BY-NC-SA 4.0)

(https://creativecommons.org/licenses/by-nc-sa/4.0/). 